\title{
USING WIRELESS TECHNOLOGY TO DEVELOP A VIRTUAL REALITY COMMAND AND CONTROL CENTRE
}

\author{
Damian Green, NeVille Stanton, Guy Walker, Paul Salmon
}

BITLAB, Brunel University, Uxbridge, Middx, UB8 3PH, England

Email: damian.green, neville.stanton, guy.walker, paul.salmon@brunel.ac.uk 01895274000

This paper investigates the applicability of wireless communication systems for use in command and control environments. Human positional data is transmitted over a wireless network. This data is then used to update a highly accurately modelled real-time 3D environment of the surroundings, with avatars positioned at the transmitted points. The data is displayed on a stereoscopic 3D screen enabling novel automatic tracking of human movement and allowing for more rapid and informed tactical decision making. The system has applicability in a variety of C4I environments, including the military and emergency services.

Keywords: Wireless Technology, Applications, Command \& Control, C4I, Human Factors

\section{$1 \quad$ INTRODUCTION}

Command, Control, Communications, Computers and Intelligence (C4I) systems gather and disseminate information around the force. This paper explores the possibility of developing a C4I system that uses currently available technology with a view to reducing friendly fire incidents. To this length, stereoscopic displays, PDAs, laptops, GPS receivers, UMTS, GPRS, 3G, UWB and various commercially available APIs are explored for their efficiacy. Processing power and usefulness of portable devices has increased greatly of late and are at a stage where they are powerful enough to transmit 
video, perform fast calculations and be used for positioning and intelligence. The aim of this paper is to investigate and outline the advantages and disadvantages to different technology and communication infrastructures.

In a C4I environment, the goal is to provide more information, more quickly and in the correct format to the elements of the force that have a need. Contemporary studies of command and control in a variety of domains show significant shortcomings in human performance [1].

The use of wireless technology to transmit positions holds the possibility for reducing friendly-fire incidents and saving lives. Friendly-fire remains a problem for even the most wired military in history, with aerial and artillery attacks the most serious. So far, 18 of the 149 fatalities suffered by U.S. and British soldiers in Gulf War II were the result of errant strikes by coalition forces. These tragic numbers appear to be an improvement over Gulf War I, which recorded 35 deaths from friendly fire - 24\% of all combat fatalities, vs. $12 \%$ in Gulf War II. But the U.S. and its allies deployed twice as many troops in the first Gulf conflict. And as the Pentagon gathers the final data from the war, the number of friendly-fire deaths could increase. Although the risks of armed conflict on a Cold Ware scale may be lower, there is increasing turbulence world-wide, with persistent mid to low-intensity threats, a trend that is likely to continue [2]. Throughout the Cold War, nations’ military forces prepared for conventional war - tank against tank, ship against ship and plane against plane. Within the past decade, the use of military force characteristically has not been state-on-state but more typically exercised in regional engagements in peacekeeping, peace enforcement and disaster relief. Armed conflict 
continues to become more complex and to require even greater precision [3]. Providing armed forces with new capabilities is essential [3].

This work’s primary purpose is to investigate the applicability of modern technology to save lives in essential services and to reduce unnecessary friendly fire incidents. This paper sets out to investigate the various technologies applicable for carrying out wireless communication of human positional data with a view to the communication of more detailed data such as audio and video. 3D data is communicated via a suitable network for the use in a C4I environment which is carefully designed around the human controller's needs. This needs-analysis is determined using human factors methods. Such methods include hierarchical task analysis [4], observational studies, verbal protocol analysis, critical decision method [5], social network analysis [6] and coordination demand analysis [7, 8].

\subsection{Friendly Fire Reduction}

In the military domain, the Battlefield Target Identification (BTID), an updated version of the Battlefield Combat Identification System (BCIS) has been developed to reduce friendly-fire incidents is. BTID uses transmitters (as did BCIS) that aim very shortwave bursts of communication data at a target just before letting go a salvo. If the target responds with the correct encrypted reply indicating that it's a friend then the gunner's sight will turn red. The system is accurate at distances of up to three miles [9]. A second program is called Individual Combat Identification System (ICIS). It comes in two parts: a specialized laser sight mounted on a rifle and a combination laser-detection device and radio transponder mounted on a soldier's helmet. When a soldier takes aim with the ICIS laser, the beam will activate the detector if the person in the sights wears 
the helmet of a U.S. soldier. The transponder will then send an encrypted signal warning the shooter not to fire. ICIS uses off-the-shelf components and weighs less than two pounds. It can run for 30 days on a single camera battery.

Soldiers who already carry 70 pounds of equipment on their backs cannot carry a heavy broadcast device powerful enough to reach fighter bombers. And those devices would need to generate a signal in a radius of 360 degrees to be able to warn not only planes but tanks and other soldiers.

Better networks for the military should make it easier to avoid friendly fire incidents. Key questions remain as to how to fit friendly-fire information into a decision that usually involves but a few seconds. The right choice can make the difference between firing upon own forces or taking fatal fire and casualties from an enemy. Friendly-fire deaths could remain an inevitability of battle for years to come.

\section{The Technology}

To provide front line forces with world class systems these have to be developed from the outset with the human operator in mind. The purpose of this research is to investigate the possibility for the wireless transmission and receiving of data to and from a person in the field (PINF) to a C4I centre. The purpose of this work is to create processes and methods which will be integrated as part of future systems.

The data transmitted from the person in the field may include Global Positioning System data (GPS), Differential GPS (DPGS), audio and video (AV). The data received back to the PINF from the CC may include GPS data of other personnel on the field, and relevant video data,

Figure 1. If GPS data of ally positions is received by the PINF, a variety of methods can 
be used for the displaying of this data on a headset, (radar notation etc).

Figure 1 illustrates example hardware technology that may be adorned by the PINF.

\subsection{Hardware Options for Mobile Nodes}

To demonstrate a proof of concept, various commercially available technologies were investigated for their applicability. It should be noted that for real-world military application, these technologies may not be secure, fast or reliable enough. The main drive of this research is to demonstrate the 3D technology; however, much of the technology which may be suitable for this kind of application is still under development. The following sub-sections suggest various commercial off the shelf (COTS) hardware configurations.

\subsubsection{Laptop and GPS Receiver with WLAN Card}

An initial proposal for the PINF hardware may include a compact laptop, for example the ultra lightweight (4.39 lbs) and compact design Sony VAIO® V505DC1 Notebook, connected to a GPS receiver via an RS232 cable, which is in turn connected to a wireless LAN card internally or through the PCMCIA slot, Figure 3a. With the option of external amplified antenna and PCMCIA option the NavRoute WAAS enabled GPS HP-60 device can be used in a notebook computer. Further investigation is needed to determine whether the GPS position can be constantly streamed from this device. The advantage to this configuration is high processing power, which may be necessary when streaming and compressing video data. The lack of compactness and the inherent superfluous technology - keyboard and LCD screen is detrimental to human movement. 


\subsubsection{PDA with inbuilt GPRS with separate GPS Device}

A second possibility requires using a more compact device, such as a Personal Digital Assistant (PDA) with inbuilt GPRS and a Bluetooth or Compact Flash GPS Receiver device plugged in, as shown in Figure 3b. PDAs currently the lack of processing power needed to perform video compression (which may be required on future prototypes). The NavRoute WAAS enabled GPS HP-600 is compatible with PDAs with a Compact Flash socket.

\subsubsection{PDA with inbuilt GPRS and GPS}

A third possibility may be through the use of a PDA with inbuilt GPS and GPRS as shown in Figure 3c. The model shown is the Garmin iQue 3600 with built in GPS PDA. Disadvantages to the possibilities identified in sections 2.1.2 and 2.1.3 include the lack of sockets for the attachment of devices such as webcams and microphones for further communication. The webcam on the PDA itself may not be sufficient because it would need to be held near the PINF's head. It is more advantageous to have the PDA separate from acquisition technology.

\subsubsection{Local Positioning Systems (LPS)}

LPS originated from the operating concepts of the global positioning system (GPS) satellite network, which takes signals from orbiting satellites to track large out-door assets, such as automobiles, trucks and railway cars.

GPS signals are too weak to penetrate walls and other obstacles and its commercial resolution is too imprecise to provide meaningful location data for indoor applications. Using these technologies and methods, LPS uses electronic tags, discreet antennas, and 
low-power radio signals to instantly locate, track, secure and inventory assets. It is ideal for stationary assets, such as large physical inventories, or for mobile assets, including people and portable equipment.

PDAs can be used in conjunction with custom-built hardware devices which receive VHF/UHF signals and triangulate. This position can then be sent using the PDAs over a WLAN or using GPRS/UMTS.

\subsubsection{WLAN Triangulation}

A laptop with WLAN card can be used in conjunction with triangulating software in an area desnsely covered with WLAN Access points (APs). Ekahau [10] have developed enhanced position determination software to allow positional information to be derived from triangulation by WLAN access points. The Ekahau Positioning Engine ${ }^{\mathrm{TM}}$ (EPE) is a positioning server that provides PC, PDA, and asset TAG location coordinates (x, y, floor) and tracking features to client applications. EPE uses a stand-alone manager application for drawing the Ekahau Tracking Rails ${ }^{\mathrm{TM}}$, recording site calibration data, tracking wireless devices on map, and statistically analyzing the positioning accuracy. Ekahau's positioning technology has an average accuracy of up to 1 meter $\left(3^{1} \frac{2}{2 t}\right)$, enabling people and asset tracking both indoors and outdoors. This opens up the possibility of WLAN networks being deployed purely for indoor tagging and tracking purposes - an application area currently dominated by proprietary tagging solutions. Ekahau Manager is used to create a positional model - A floor-plan or map image (BMP, JPG, PNG) is opened and Ekahau Tracking Rails are drawn on the map to increase positioning accuracy and stability. The Positioning Model is created by moving around the area while clicking the map to record sample points containing received signal 
strength intensity (RSSI) samples. No information about the access point locations is required. Investigation is underway to investigate if improved methods of triangulation are possible using WLAN APs. A dense deployment of APS across one third of Brunel University’s campus is underway such that at any position there is always three overlapping radio signals, Figure 7.

\subsubsection{C4I Communication Hardware}

If GPRS is used as the communication medium, at the CC end, a GPRS equipped device with contract is all that is required, and the CC connects to the mobile device through a standard network connection. If the CC is non-mobile and network capable then this is no problem. The $\mathrm{O}_{2}$ company offers such an ISP connection. However if the CC is mobile yet is still in the vicinity of mobile phone base-stations then a GPRS card and dedicated phone line is necessary. GPRS however is too slow for the transmission of video data. Tthe maximum bandwidth being around $115 \mathrm{kbs}$, if only one client is connected to the GPRS basestation. For the CC environment, a large Stewart stereo wall was used (Figure 4) [11]. If WLAN is used as the transmission medium, a connection to the Internet is all that is needed at the CC end.

\subsubsection{Hardware Options Discussion}

Some of the PDAs described have GPRS transmission capability. In absence of a WLAN, this may be a viable communication medium. The emerging standard of Universal Mobile Telecommunications System (UMTS) may be more appropriate. UMTS represents an evolution in terms of services and data speeds from today's "second generation" mobile networks. UMTS is the natural evolutionary choice for operators of 
GSM networks, which currently represent a customer base of more than 850 million end users in 195 countries and represents over 70\% of today's digital wireless market The Ekahau solution sounds like a good approach for indoor triangulation or in cases where WLAN coverage is great and GPS is unavailable. The accuracy is not enormous however and it requires significant pre-calibration. It is difficult to imagine how the deployment of WLAN devices and the creation of positional models would be useful for anything other than the development of aesthetically pleasing prototypes for a system which would later use alternative positioning technology.

PDAs using the Pocket PC operating system (OS) capable of GPRS are available on most of the HP modern HP series; Jornada, iPaq 5500, 5550 and so on. Combined with a GPS device , a compact portable wireless programmable GPS solution can be realised. This combination is shown in Figure 3. However if the device is in the vicinity of a WLAN then a PDA which has integrated WLAN 802.11b such as the HP iPAQ 5500 would allow free transmission of position in areas with WLAN coverage, such as the environment mentioned. One which requires a plug-in WLAN is unsatisfactory because there is then no space to include a GPS receiver.

Outdoors, GPS devices are easily hampered by adverse weather conditions - cloud cover and so on.

Bluetooth wireless technology is designed to replace cables between cell phones, laptops, and other computing and communication devices within a 10-meter range. As such it is not useful for the transmission of GPS data to a remote CC, although it may have useful applications for local PINF communications (comms). Wi-Fi is wireless network; it provides an extension or replacement of wired networks for dozens of computing 
devices.

There is an increasing number of phones released with 3G capabilities, although these do not currently have built in Wi-FI. A similar technique has been developed for transmission of video data as described by Hunaiti et al [12] in their navigation system for blind pedestrians. 3G base stations are currently not widespread enough for reliable transmission of vidoe. In the areas where $3 G$ base stations are deployed, usage is often high, so this tehcnology cannot yet be relised upon for the application being developed. Pocket MSN Messenger would appear to be a viable commerical off-the-shelf (COTS) solution for video transmission. At present it does not send video data. It has been expressed by those with long standing experience that a very high proportion of end-users of military systems, and those in the Navy and Army are already familiar with PCs, Microsoft Office and email, so the development of software which behaves in as similar a way as these is more preferable than complicated proprietary solutions. To this end, COTS technology is sought in the first instance.

PocketTV is a software client which plays streaming video from a web based source. In order to stream real-time video with PocketTV a device must be equipped with high-bandwidth (>=500 Kbit/sec) wireless Internet access. No extra memory is needed no the PDA, the .mpg video will just be streamed. At the C4I end, a web server must be configured to enable pocketTV streaming. PocketTV is capable of streaming from standard HTTP/1.1 servers such as Apache. It is more likely that the client devices wlil not need to receive video data, but instead isntructons and position information,. It is more important that the C4I system receive video data. 


\subsection{On Developing a GPS Receiving PDA application}

A variety of PDAs were acquired for development purposes, including the HP Jornada which has GPRS and a CF slot for a GPS device, as well as the later numerous iPAQ 5550s which feature both WLAN capability and a CF slot for GPS receivers. The Microsoft Visual Studio .NET Application Wizard was used to create a Visual Basic Smart Device Application. The .NET Compact Framework is a hardware-independent environment which can run programs on resource-constrained computing devices, encompassing personal data assistants (PDAs) such as the Pocket PC, mobile phones, settop boxes, automotive computing devices, and custom-designed embedded devices built with the Windows CE .NET operating system. The number and members of classes differ from what is available for developing desktop applications. Fewer classes are available for devices using the .NET Compact Framework (for example, Web forms, remoting, messaging, management, and printing are not supported), and the enumeration of classes typically differs among platforms. PDAs currently have a restriction on the number of devices which can currently be plugged into the compact flash socket. If a GPS device is already plugged in then there would be no space for a webcam. There are emerging webcam technologies which connect directly to WLAN access points streaming video. These could be used in conjunction with PDAs.

Tactical commands are issued as coded instructions, which in turn would play concatenated pre-recorded audio files stored on the PDA, such as „Move team North 10 clicks“. This reduces bandwidth usage significantly and also means that the soldier does not need to be physically looking at or interacting with the PDA, and as a result is more aware of the battlefield. 


\subsubsection{Receiving GPS}

Programs which allow communication with GPS devices were investigated. An example VB application which communicates between a PDA and GPS device is to be found on the MSDN library [13]. However it requires the use of a COMM control. Visual Studio .NET does not have this control as standard and Visual Studio 6, or VB 6.0 needs to be used, or through a technique known as Interop. Interop is hidden from the programmer by Visual Studio so that it looks like another .NET control. Using Interop adds an unneeded level of complexity and performance penalty to programs. An early prototype of a client application which uses GPS positioning and a radar type interface is shown in the photograph in Figure 5.

There are a wide number of GPS devices available for PDAs and laptops. Both Bluetooth or Compact Flash (CF) devices are available. If WLAN is used for transmission then there is the possibility that the Bluetooth GPS receiver will interfere becasue they both share the same frequency range. Bluetooth also consumes a lot of power on the PDA side, but there is the advantage that the GPS device wlil not be draining lots of power frmo the PDA's battery. Having a seperate battery for the Bluetooth GPS device would increase the complexity when it comes to charging the devices. In the military domain, anything that can increase simplicity is highly important. GPS devices themselves, when plugged into PDAs can drain the battery very quickly - in a matter of minutes. This is not practical for military applications and either larger batteries or lower power consumption receivers need to be sought. 


\section{$2.3 \mathrm{C} 4 \mathrm{I}$}

To develop a C4I application which communicates with wireless technology and displays this data in 3D stereo in real-time, careful consideration needs to go to the choice of software platform.

Visual Basic provides rapid application development (RAD) but is lacking in fast realtime 3D graphical display. The use of the OpenInventor (OIV) IVF interface library for gluing Inventor to Microsoft Foundation Classes (MFC) works well using the $\mathrm{C}++$ platform [14]. The purpose of the IVF class library is to simplify integration of functionality provided by the Open Inventor Win32 Classes with MFC-based applications. IVF builds upon the application framework provided by MFC to deliver the additional functionality of Open Inventor in a manner familiar to MFC developers. A "socket" is an endpoint of communication: an object through which an application communicates with other Windows Sockets applications across a network. MFC contains two different classes for communicating via sockets (WinSock)[15]. TGS's OIV allows for rapid development through its Visual Studio AppWizard extensions. It also allows for easy stereo extensibility to applications, which is necessary for C4I visualization on the screen shown in Figure 4.

A preliminary application has been developed which uses the aforementioned technology as shown in Figure 6. The application imports a 3D model in the VRML format (.iv) of the environment over which data is wirelessly communicated. This has been modelled from AutoCAD survey data from the estates department of the university and using the theodolite measured point and line data to extrude areas of grass as a baseline for the modelling of buildings. Some buildings have been left as wireframe extrusions of survey 
data to show the different representational schemes possible to the commander. Further investigations will determine the most useful representation of the situation. A schematic diagram of the software configuration is shown in Figure. 2. Initial tests of the software have realized impressive update speeds, even on a Alienware $3.2 \mathrm{GHz}, 2 \mathrm{~GB}$ RAM desktop machine.

\subsection{Human Factors}

Rather than suggest that one form of representation suits all types of task, our proposal is to investigate the appropriateness of each representation for each aspect of the scenario and the decisions being made. It is anticipated that more strategic, tactical and operational tasks might require different types of representation. For example, formulation of strategies might best be supported by more abstract, goal-oriented displays of information. Operational tasks might be better supported by a physical analogue representation of the real world. Tactical tasks might require both sorts of representation, or a merging of the two forms of data.

Matching the team role to the representation might be an overly constrictive approach to interface design, but allowing members of the team to have access to different representations of the scenario relevant to the task that they are controlling may be more fruitful.

Operational testing of the prototype environments will be undertaken within an experimental paradigm. First, we need to judge exactly what performance gains can be made over conventional C4I methods. Second, we need to discover what process improvements there are to be made with these new forms of C4I technology. There may 
be additional performance improvements to be realized by using the technology to perform tasks more effectively.

General research questions into multiple agent-based computing interaction needs to be addressed It is inevitable that network enabled computing will lead to new forms of interaction. As the task-artefact cycle products, new artefacts produce new tasks and new tasks produce new artefacts. Network enabled technologies are likely to have a considerable social component to the interaction, supporting human-human interaction via computing.

\section{CONCLUSION}

Robinson et al. write that for sound legal and operational reasons in our pluralistic society, we shall require an audit trail of operational decisions and consequences [2]. By virtue of the fact that all movements are streamed over a network in the approach described, human paths can be recorded and later replayed with a minimum of effort in a 3D environment. This allows for a better criticism of tactics employed with the aim of improving future tactics. This could provide useful input to movement behavioral models

such as those developed by Reece [16]. This research project is still in its early stages and with the intended Human factors investigations; detailed testing of these technologies will likely unfold some interesting results [8].

The accuracy of GPS signal, urban density, weather conditions and wireless LAN coverage of the environment are all limiting factors to the approach's usefulness. In a new, unmodelled and unwired environment the full potential of this technology would not be realized to the detail shown. Although it is envisaged that the use of photogrammetric approaches could be employed. For example, an aircraft could be flown 
at a constant altitude over the environment taking a series of images; the images would then use feature matching to create a coarse point cloud.

Future work with VR headsets and haptic devices for more immersive visualization and interactivity both at the CC and at the PINF side is a topic set to be investigated.

There are many potential applications of the technology in civil, military and emergency services C4I. Any domain that has a central command and distributed workforce has the potential to benefit from the insights resulting from this research.

The precise nature of the transmission and communicational technology is not yet decided but decisions made on the use of the airwaves for transmission methods such as UWB [17] will affect decision making.

This project is in its infancy but our prime objective is to investigate the use of technology to reduce friendly-fire incidents and thus saving lives. The main method of doing this is by using technology to pinpoint ally positions and relay this to them in an informative and fast way.

\section{Acknowledgement}

This research was made possible by funds provided by the UK Ministry of Defence through DSTL to support the Human Factors Integration Defence Technology Centre.

\section{References}

[1] Smith J (2003) Assesment of C4i Systems Defence Management Journal 23:74-76

[2] Robinson P and Pickard L C I (2003) The UK approach to future Command and Inform (C4ISR) Journal of Defence Science 3:179-186

[3] Hubbard M and Fentone C G (2003) Non-lethal capabilities and the road ahead Journal of Defence Science 3:210-

214 
[4] Annett J, Duncan K D, Stammers R B and Gray M J (1971) In:Task analysis Department of Employment Training Information Paper No. 6 London, UK: Her Majesty's Stationary Office (HMSO).

[5] Klein G A, Calderwood R and MacGregor D (1989) Critical decision method of eliciting knowledge IEEE

Transactions on Systems, Man and Cybernetics 462-472

[6] Driskell J E and B M (2004) In:Social Network Analysis Handbook of Human Factors methods Boca Raton: CRC Press.

[7] Burke (2004) In:Team Task Analysis Handbook of Human Factors Methods Boca Raton: CRC Press.

[8] Stanton N A, Hedge A, Brookhuis K, Salas E and Hendrick H (2004) In:The Handbook of Human Factors and

Ergonomics. London: Taylor and Francis

[9] Science F o M (2003) Battlefield Combat Identification System (BCIS) http://www.fas.org/man/dod-

101/sys/land/bcis.htm

[10] Ekahau (2003) Triangulation Software http://www.ekahau.com

[11] Corporation S F S (2003) Stewart Screens http://www.stewartfilm.com

[12] Hunaiti Z, Garaj V, Balachandran W and Cecelja F (2004)An Assesment of GSM/GPRS Link in a Navigation System for Visually Impaired Pedestrians In:14th International Conference on Electronics, Communications and Computers (CONIELECOMP'04)

[13] MSDN (2003) Migrating a GPS App from the Desktop to eMbedded Visual Basic 3.0

http://msdn.microsoft.com/msdnmag/issues/01/01/GPS/default.aspx

[14] TGS (2003) TGS Open Inventor http://www.tgs.com

[15] Microsoft (2003) MSDN http://msdn.microsoft.com

[16] Reece D A (2003) Movement Behavior for Soldier Agents on a Virtual Battlefield Presence (Teleoperators and Virtual Environments) 4:387 - 410

[17] O'Brien D (2004) Radio Freedom New Scientist 2444:28-31

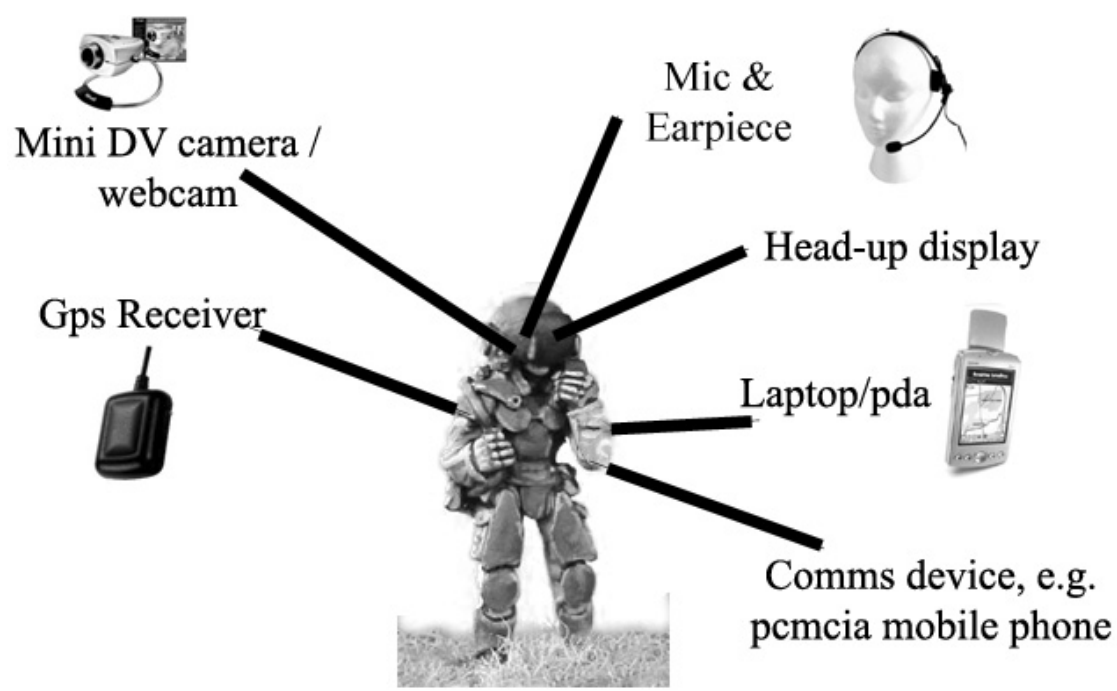

Figure 1. PINF Hardware 


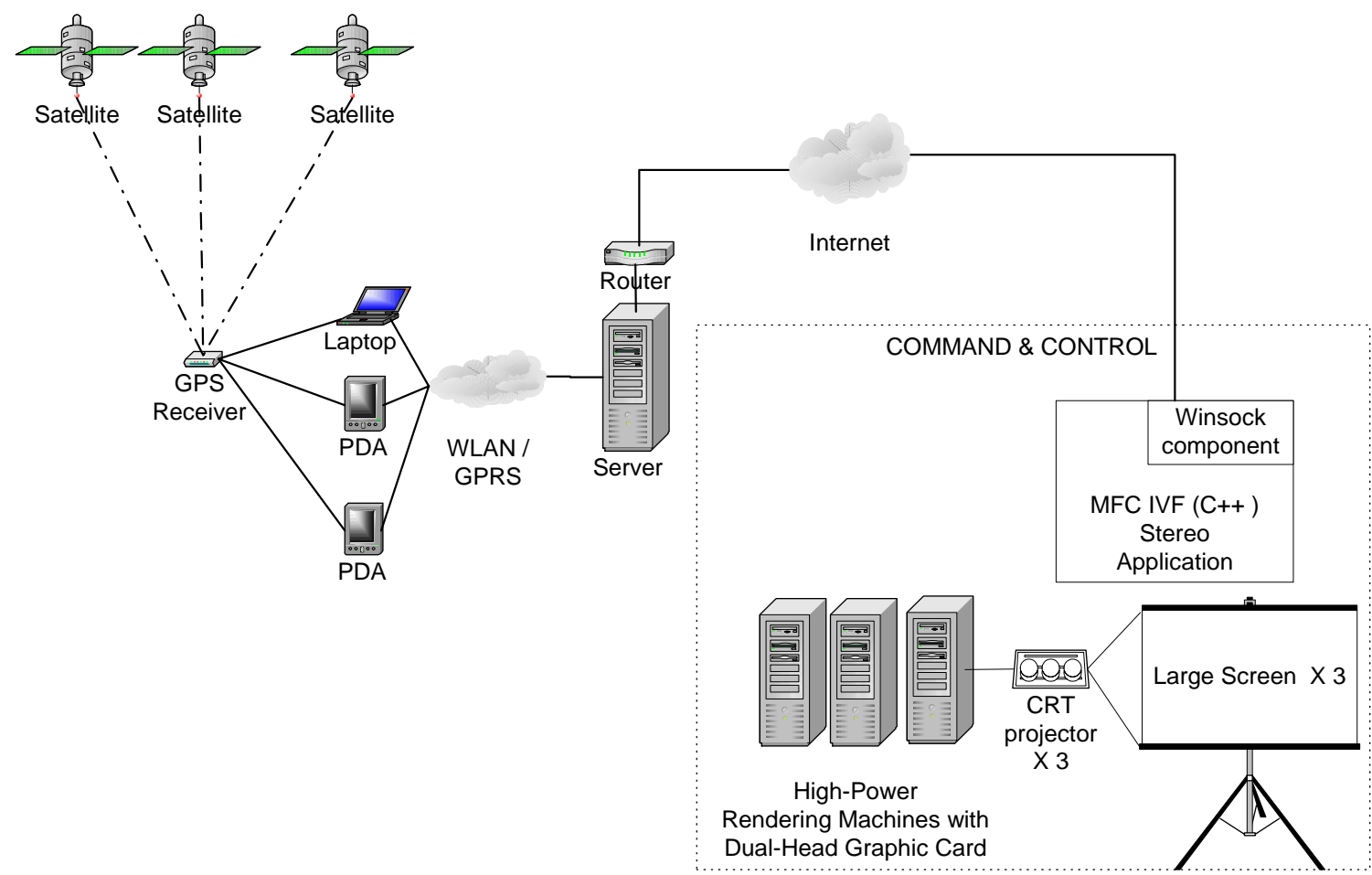

Figure. 2. Overall System Concept. 

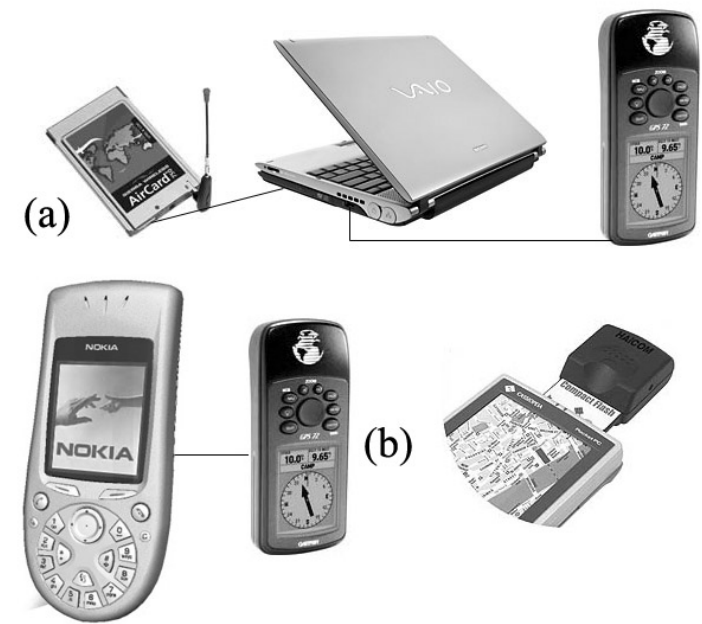

(b)
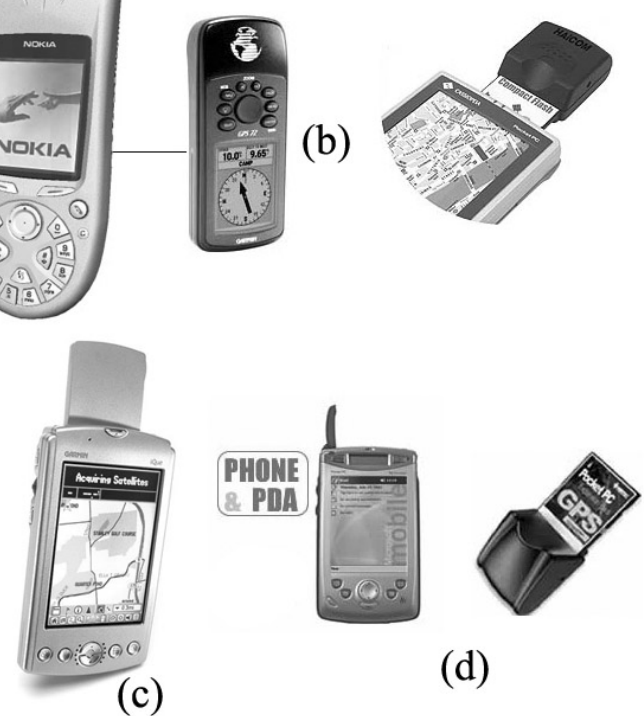

(d)

(c)

Figure 3. Candidate Hardware Configurations for Client Position Communication Device a) Compact PC with GPS receiver connected via GPRS Wireless PCMCIA card. b) PDAs with GPS. c) PDA with inbuilt GPS. d) PDA with GPRS and CF GPS. 


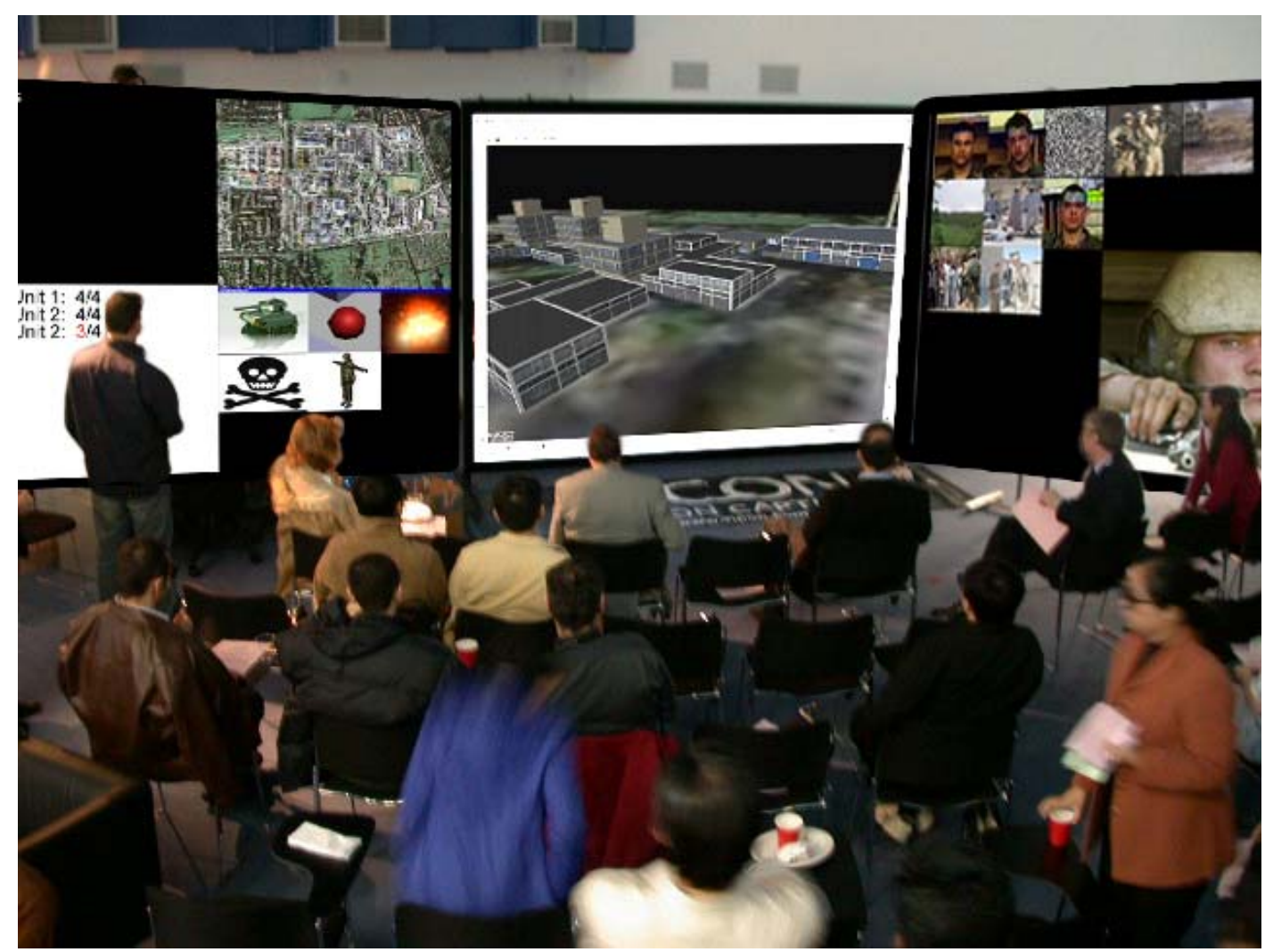

Figure 4. Command and Control Centre shown on a stereo Stewart Screen at the Bitlab, Brunel University, UK

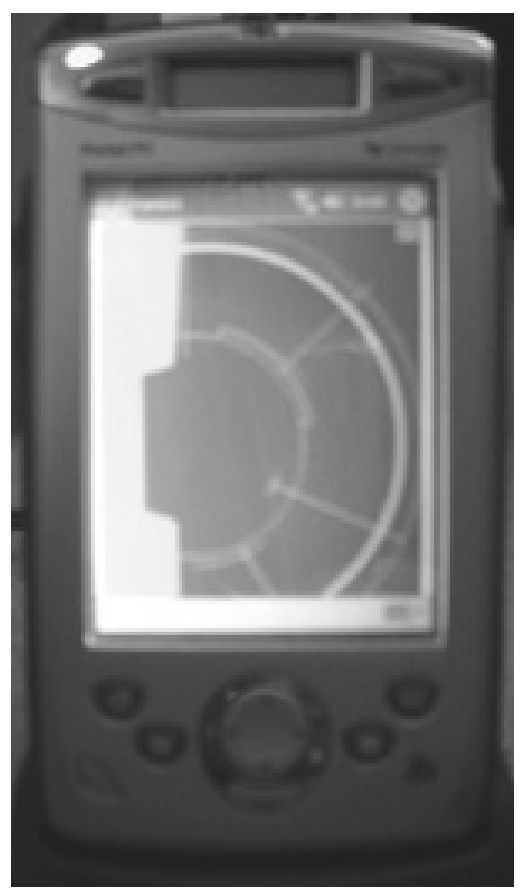

Figure 5. An Early GPS Radar Application Running on an HP Jornada PDA. 


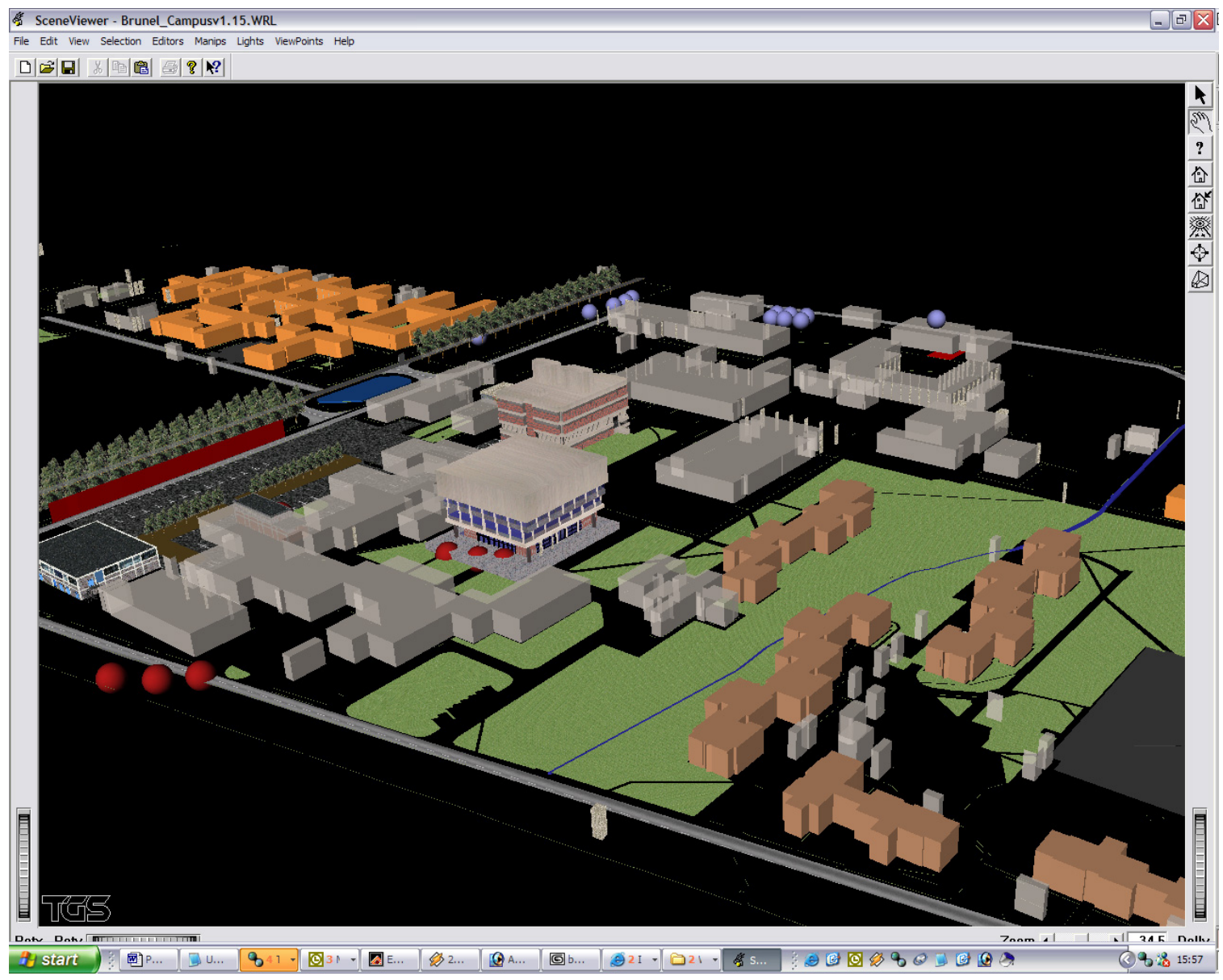

Figure 6. Proprietary OpenInventor Application Showing 3D Model of Environment Used for Wireless Positional Communication. 


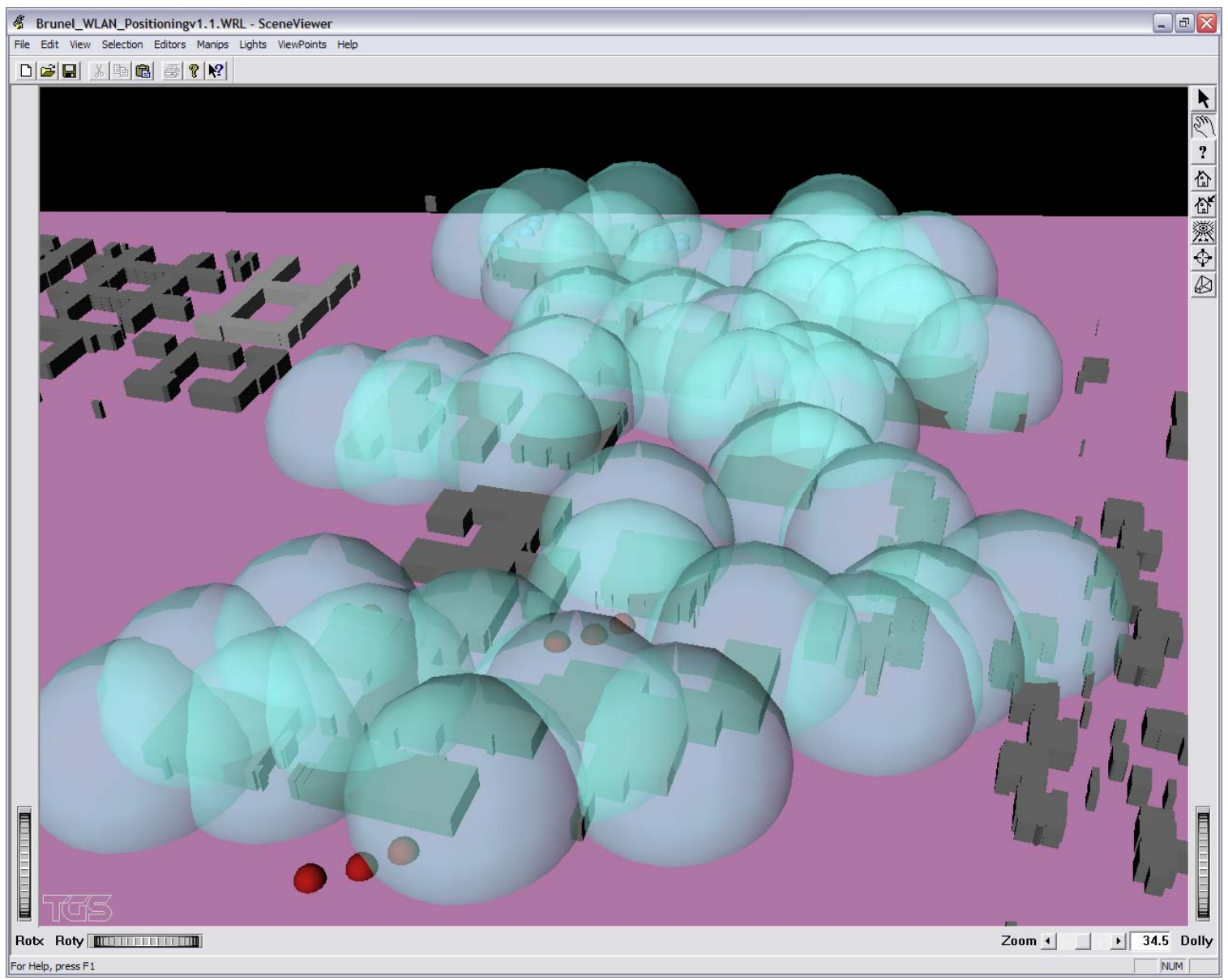

Figure 7. WLAN AP rollout on Brunel Campus. 